\title{
Tra beatitudine ed im-potere: note sull'avvenire del biopolitico (Deleuze vs Derrida)
}

\author{
Between beatitude and impouvoir: notes about biopolitic's future \\ (Deleuze vs Derrida)
}

Pietro Lembo ${ }^{1}$

\begin{abstract}
: partendo dal pensiero foucaultiano, ed in particolare dalla tesi secondo cui la rottura epistemologica tra sovranità e biopolitica avrebbe svelato la consustanzialità tra potere e vita, il presente contributo intende rintracciare nelle filosofie francesi della differenza, ossia nel pensiero di Deleuze e di Derrida, due filosofie della vita che, nel pensare il nesso tra vita e potere in modo antitetico, risultano imprescindibili qualora si voglia riflettere circa l'avvenire della bipolitica. Parole chiave: vita, morte, desiderio, beatitudine, impotere.
\end{abstract}

\begin{abstract}
: recovering the foucaultian's thought, and particularly the argument that epistemological rupture between soverignty and biopolitics have disclosed the relationship between power and life, the present contribution aims to consider the french philosophy of difference - i.e. Deleuze's and Derrida's thought - as two philosophy of life that, thinking in opposite way the connection between life and power, result important to analyse the future of biopolitics. Keywords: life, death, desire, beatitude, impouvoir
\end{abstract}

\section{I sentieri interrotti di Michel Foucault}

Secondo la nota analisi di Michel Foucault, la biopolitica indica una configurazione del potere che ha preso il posto della sovranità: mentre quest'ultima si esercitava lasciando vivere e facendo morire, la biopolitica si caratterizza per il fatto di lasciare morire e fare vivere, vale a dire per il fatto di legiferare in materia di corpi, assumendo i tratti di un inedito sistema di intervento sulla vita, sulla scorza biologica dell'esistenza ${ }^{2}$.

\footnotetext{
${ }^{1}$ Professor titular da Universidade Presbiteriana Mackenzie. Professor colaborador da Faculdade de Direito da Universidade de São Paulo. E-mail:<pietrol@live.it>

${ }^{2}$ Stando alla ricostruzione foucaultiana, questo passaggio dalla sovranità alla biopolitica sarebbe avvenuto nella modernità alla stregua di un cambio di paradigma: "quel che si potrebbe chiamare la «soglia di modernità biologica» di una società si
} 
Frutto della secolarizzazione, che ne ha eroso il nucleo teologico-politico e trascendente, detto potere si è disseminato nella società capillarizzandosi, pervadendo tutti gli anfratti dell'esistenza, ma, perciò stesso, frantumandosi e smembrandosi in una molteplicità di relazioni di forza, in una molteplicità di micro-lotte intestine.

Proprio per questo, detto sistema di controllo delle vite e dei corpi sembra serbare in sé le possibilità del proprio smontaggio. Le suddette micro-lotte intestine sembrano infatti mettere in luce una pista presente in Foucault ma non sufficientemente sviluppata: il fatto che la vita non è semplicemente l'oggetto del potere, bensì anche il suo soggetto, il quale, come tale, è nelle condizioni di resistergli opponendovisi. Come dice Esposito alludendo proprio al pensiero di Foucault:

L'autore sembra divaricarsi in direzioni divergenti che chiamano in causa [...] due nozioni, fin dall'inizio implicate nel concetto di bíos, ma situate agli estremi della sua estensione semantica: quella di soggettivazione e quella di morte. Entrambe - rispetto alla vita - costituiscono più che due possibilità. Sono allo stesso tempo la sua forma e il suo sfondo, la sua origine e la sua destinazione. Ma in ogni caso secondo una divergenza che sembra non ammettere mediazioni: o l'una o l'altra. O la biopolitica produce soggettività o produce morte. $\mathrm{O}$ rende il soggetto il proprio oggetto o lo oggettiva definitivamente. O è politica della vita o sulla vita ${ }^{3}$.

Sebbene interna al discorso di Foucault, la politica della vita - e quindi la riflessione attorno al potere della vita - è suscettibile di essere considerata alla stregua di un sentiero interrotto della filosofia foucaultiana, la quale, dopo la parentesi biopolitica, ha rivolto la propria attenzione ad altri temi ${ }^{4}$.

\footnotetext{
colloca nel momento in cui la specie entra come posta in gioco nelle sue strategie politiche. Per millenni l'uomo è rimasto quel che era per Aristotele: un animale vivente ed inoltre capace di esistenza politica; l'uomo moderno è un animale nella cui politica è in questione la sua vita di essere vivente" (FOUCAULT, M. Foucault, La volontà di sapere. Storia della sessualità 1. Milano: Feltrinelli, 2011, p. 127). In merito a tale ricostruzione, Derrida non ha mancato di mostrare una certa reticenza che andrebbe indagata proprio all'interno del rapporto tra Derrida e Deleuze: tralasciando lo studio iniziale su La storia della follia, si veda, ad esempio, DERRIDA, J. «Al di là del principio di potere». In: DERRIDA, J. «Essere giusti con Freud». La storia della follia nell'età della psicoanalisi. Milano: Raffaello Cortina, 1994, p. 105; DERRIDA, J., La Bestia e il sovrano I (20012002). Milano: Jaca Book, 2009, pp. 379-429.

${ }^{3}$ ESPOSITO, R. Bíos. Biopolitica e filosofia. Torino: Einaudi, 2004, p. 25.

${ }^{4}$ In particolare al tema della cura di sé, cfr. FOUCAULT, M. Storia della sessualità. Vol. 3: La cura di sé. Milano: Feltrinelli, 2001.
} 
È per questo che l'avvenire della riflessione biopolitica sembra necessitare che ci si collochi nel punto in cui Foucault ha interrotto il proprio sentiero, di modo da comprendere in che direzione prolungarlo.

Molteplici sono le vie che si possono diramare a partire da questa prosecuzione, ma siamo convinti che ve ne siano due particolarmente pregnanti nell'ambito della filosofia francese del Novecento: quelle di Deleuze e di Derrida. Entrambi infatti - probabilmente in modo involontario - hanno prolungato il sentiero interrotto di Foucault, portandolo verso due conclusioni inverse. Nel caso deleuziano, la vita assurge a potenza capace di eccedere tutti i poteri costituiti: è questo ciò che egli chiama la beatitudine della vita. Nel caso derridiano, la vita viene concepita come una strutturale non appartenenza a sé - l'exappropriazione - che disvela l'im-potere al cuore di tutti i poteri.

Ebbene, la scelta in favore di uno di questi due paradigmi ${ }^{5}$ - la beatitudine o l'im-potere - non è forse tale da decidere l'avvenire della biopolitica?

\section{Biopolitica e beatitudine in Gilles Deleuze}

Cominciamo anzitutto col ripercorrere le tappe che hanno permesso a Deleuze di elaborare un pensiero del vivente dalle ricadute importanti sul rapporto tra vita e politica ${ }^{6}$.

Si tratta di un pensiero che il filosofo di Vincennes ha elaborato adoperando molte delle categorie sviluppate in seno all'epistemologia francese anti-kantiana ${ }^{7}$ di cui Canguilhem e Simondon sono i nomi di spicco.

Uno dei meriti forse più importanti di Canguilhem è quello di aver ripensato la relazione tra vita e norma. Servendosi in particolare del pensiero di Cavaillés, il medico-filosofo professore alla Sorbonne, ha

\footnotetext{
${ }^{5}$ Per un recente confronto circa la filosofia di questi due pensatori, cfr. E. BISET, E. «Ontología de la diferencia». In BISET, E., FARRÁN R., Ontología política, Buenos Aires: Imago Mundi, 2010; JABRE, E. «Le collectif commence seul, c'est-à-dire à plusieurs. Une tentative de compliquer le jeu des oppositions politiques», Chimères, 81, (2013).

${ }^{6}$ È di questo avviso Esposito, cfr. ESPOSITO, R. Bíos, cit.

${ }^{7}$ A dire il vero il pensiero filosofico del vivente (soprattutto di matrice deleuziana) è stato influenzato anche da altri pensatori di fondamentale importanza, tra i quali spiccano, indubbiamente, Spinoza, Nietzsche e Bergson; se, in questa sede, non si è cominciato da questi è, evidentemente, per ragioni di spazio, talché si è scelto di partire da quegli autori le cui ricadute in termini di biopolitica sono, per così dire, più esplicite. Ad ogni modo, per una disamina della storia della filosofia in ordine al problema della vita e della sua potenzialità, cfr. CICCARELLI, R. Immanenza. Filosofia, diritto e politica della vita dal XIX al XX secolo. Bologna: Il Mulino, 2008.
} 
mostrato che la norma - lungi dall'essere una forma trascendente capace di decidere il vivente entro un preciso schema ermeneutico-politico - è, piuttosto, il risultato del flusso immanente della vita. Più specificamente, la reinterpretazione del DNA in termini di codificazione linguisticosimbolica, ha permesso a Canguilhem di mostrare che la vita consiste nell'alterazione che risulta dagli errori di trascrizione del codice genetico, sia nel passaggio generazionale, sia nell'interazione con l'ambiente. Ad essere completamente stravolto è, quindi, sia il concetto di normalità, sia quello di patologia. Se la norma-di-vita è un concetto immanente al vivente, il patologico non è l'alterazione del normale, bensì la cifra dell'evoluzione biologica dovuta agli errori di trascrizione del DNA. Questi stessi errori sollecitano continui processi di ri-codificazione dell'esistenza che determinano la norma-di-vita. L'immanenza tra vita e norma, perciò, assurge a intreccio potenziale, poietico e generativo in virtù del quale l'individuo è "sempre al di là di se stesso": vivere significa eccedere continuamente le forme nelle norme, le quali, invece che normalizzare l'esistenza, ne amplificano grandemente le potenzialità.

Venendo a Simondon, il contributo di quest'autore alla nostra questione è stato, indubbiamente, l'elaborazione del concetto d'individuazione, il quale, in polemica con l'ilomorfismo aristotelico, ha permesso di esplicitare il fatto che l'individuo sia in realtà un'entità costantemente in rapporto con un sistema - campo pre-individuale - con cui scambia energia in modo potenziale. Rispetto a tale campo, caratterizzato dal fatto di essere virtuale, l'individuo attualizza la virtualità potenziale determinandola in modo specifico. Al contempo, il virtuale eccede l'individualità caricandola di possibilità inespresse e, tuttavia, sempre soggiacenti. Posto ciò, emerge chiaramente il significato dell'individuale; esso è "potenza individuata" 9 È, riprendendo Spinoza, solo uno degli infiniti modi di determinazione della potenza. Il preindividuale, rinviando alla physis ${ }^{10}$ presocratica, in specie all'apeiron di Anassimandro, costituisce la base virtuale con cui eccedere le opposizioni binarie tra uomo e animale, individuo e società, natura e artificio. Ogni

\footnotetext{
${ }^{8}$ ESPOSITO, R. Bíos, cit., p. 208.

9 VIRNO, P. «Moltitudine e principio di individuazione». In: SIMONDON, G. L’individuazione psichica e collettiva, Roma: DeriveApprodi, 2006, p. 276.

${ }^{10}$ Per ulteriori approfondimenti in merito, cfr. COMBES, M. «Prefazione». In: SIMONDON, G. L'individuazione psichica e collettiva, cit., p. 9 .
} 
forma di esistenza, quindi, non è più racchiudibile entro una precisa determinazione; piuttosto si dispiega nella superficie dell'individuazione (o piano d'immanenza ${ }^{11}$ ) in cui l'attuale è potenziato dal virtuale.

È a partire da questo retroterra canguilhemiano-simondoniano che Deleuze, sviluppa un inedito pensiero della vita.

Due le parole chiave deleuziane da menzionare in proposito: different-z-iazione e contro-effettuazione dell'evento. La prima, risalente a Differenza e ripetizione ${ }^{12}$, implica la correlazione fra differen-z-iazione (attualizzazione) e differen-t-iazione (virtualizzazione) tramite cui eccedere ogni organizzazione attuale della vita. Come si legge in Differenza e ripetizione:

Denominiamo differentiazione la determinazione del contenuto virtuale dell'idea, e differenziazione l'attualizzazione di questa virtualità in specie e parti distinte [...]. Il primo processo si confonde con la descrizione di una positività pura, nella modalità del problema in cui sono assegnati rapporti e punti, posti e funzioni, posizioni e soglie differenziali, che escludono ogni determinazione negativa e trovano la loro fonte in elementi di affermazione generici o produttori. Il secondo processo si confonde con la produzione di affermazioni generate finite, che vertono sui tempi attuali che occupano quei posti e quelle posizioni, sulle relazioni reali che incarnano quei rapporti e quelle funzioni ${ }^{13}$.

Se dunque la differenziazione del vivente corrisponde al processo di attualizzazione, e quindi alla determinazione di qualcosa di potenziale nell'atto della sua concrezione, d'altronde, quello stesso vivente resta pur sempre differentiato, caratterizzato da una certa virtualità che gli permette di eccedere la sua forma: la virtualità "è la stessa cosa dell'attualità, o individuazione, ma resta fuori da essa” ${ }^{14}$. In questo quadro la vita appare

\footnotetext{
${ }^{11}$ Come mostra Ciccarelli, le tracce embrionali della logica immanentista si trovano già nel pensiero neoplatonico, il quale ne fece uso al fine di tracciare una differenza tra l'Uno (trascendente) e le emanazioni che da questo principio trascendente derivano. In seguito è stato il cristianesimo paolino ad appropriarsi del paradigma immanentista tramite la figura del mediatore cristiano - perno della mondanizzazione della trascendenza. Con la modernità, invece, si assiste all'interiorizzazione dell'immanenza entro la coscienza trascendentale di Cartesio, Kant e Husserl. Per quanto riguarda gli ascendenti più diretti nel periodo contemporaneo, invece, vanno ricercati in ambito francese, in una certa tradizione dello spiritualismo (in particolare Bergson), nella fenomenologia (soprattutto Merleau-Ponty), in una ripresa dell'ontologia spinoziana e, infine, nell'epistemologia anti-kantiana. Per ulteriori approfondimenti sul tema si rinvia a: CICCARELLI, R. Immanenza, cit., pp. 50-52.

${ }^{12}$ DELEUZE, G. Differenza e ripetizione. Milano: Raffaello Cortina, 1997, pp. 267-268.

${ }^{13}$ Ibidem.

${ }^{14}$ CICCARELLI, R. Immanenza, cit., p. 175.
} 
come una singolarità che, lungi dall'essere radicata nell'empiricità della propria individualità, si moltiplica nelle sue possibilità pre-individuali. È in questo senso che Deleuze sottolinea che l'uomo vive al limite della propria vita $^{15}$, limite caratterizzato dalla possibilità di essere sempre superato.

L'altro concetto, centrale invece in Logica del senso, implica che si consideri ogni evento della vita in modo duplice, e ciò in conseguenza del fatto che l'attualità è sempre contro-effettuabile in rapporto al campo virtuale che tutto sottende. Contro-effettuare l'evento significa "liberare, all'interno di ciò che accade la potenza evenemenziale in esso racchiusa”" significa stimolare il libero flusso vitale contro la coartazione delle forme.

Dimorando in esso, e quindi "elidendo ogni forma di trascendenza"17, questa logica sollecita il brulichio pre-individuale-virtuale, culminando in quella che Deleuze definisce "una sorta di beatitudine"18.

Ma come intendere una tale beatitudine? Questo termine, che rinvia alla dottrina dell'univocità ontologica di matrice scotista ${ }^{19}$, indica una condizione precedente alle forme che, nel corso dell'evoluzione biologica, hanno presieduto al processo di specificazione delle varie specie viventi. Molteplici sono gli esempi di questa beatitudine.

Tra essi spicca senza dubbio quell'esperienza del corpo che Artaud definiva, appunto, corpo-senza-organi: chiamando in causa le esperienze corporali precedenti alla differenziazione organica (un esempio in tal senso è l'embrione), detto corpo sarebbe tale da riportare alla luce le tracce di un vivere non ancora organizzato e configurato nelle forme del corpo organico.

Un altro esempio della beatitudine deleuziana è rintracciabile in quella logica del desiderio che Deleuze tratteggia, insieme allo psicoanalista Félix Guattari, nell'ambito dell'Anti-Edipo. Deleuze e Guattari, in quest'opera, propongono una nozione di desiderio, per così dire anti-psicoanalitica. Stando ai due pensatori, infatti, la psicoanalisi, con la sua tesi del complesso edipico - secondo cui il piccolo d'uomo desidererebbe giacere con la madre ed uccidere il padre, salvo poi fare retromarcia identificandosi con il padre e

\footnotetext{
${ }^{15}$ DELEUZE, G, Logica del senso. Milano: La Feltrinelli, 2005, p. 97.

${ }^{16}$ ESPOSITO, R. Terza persona. Politica della vita e filosofia dell'impersonale. Torino: Einaudi, 2007, p. 174.

${ }^{17}$ Ivi, p. 179 .

${ }^{18}$ DELEUZE, G. L’immanenza: una vita.... Milano: Mimesis, 2010, p. 5.

${ }^{19}$ Svelando che l'essere si dice in un unico e medesimo modo per i differenti enti di cui si compone (univocità), Scoto, nota Deleuze, avrebbe fornito i presupposti con i quali decostruire ogni tentativo di gerarchizzazione ontologica; per ulteriori approfondimenti cfr. DELEUZE, G. Differenza e ripetizione, cit., pp. 52-6o.
} 
metonimizzando il desiderio materno - culminerebbe in una concezione nichilistica del desiderio. Un desiderio metonimico, difatti, è un desiderio che ha da sempre rinunciato alla propria meta originaria (madre), è un desiderio che si sperimenta sulla base dell'auto-interdizione della propria meta (incesto), è, in ultima istanza, un desiderio afflitto da una mancanza incolmabile ed insaturabile che Lacan definì con il termine di reale impossibile. Contrariamente a ciò, Deleuze e Guattari sostengono con fermezza che "il desiderio non manca di nulla" ${ }^{20}$, ragion per cui propongono di situare il desiderio nel posto vuoto del reale, approdando ad una conclusione inversa rispetto a quella lacaniana: "il reale non è impossibile, nel reale anzi, tutto è possibile, tutto diventa possibile"21.

La logica che Deleuze e Guattari seguono nel proporre la loro nozione di desiderio è molto simile a quella che ha spinto a riesumare la nozione di corpo-senza-organi: così come quest'ultimo sarebbe tale da rievocare esperienze corporali antecedenti alla differenziazione organica, allo stesso modo la logica anti-edipica dovrebbe comportare una regressione ad uno stadio del desiderio non ancora simbolizzato, e, quindi, negativizzato.

Radicalizzando ulteriormente, si potrebbe dire che il corpo-senzaorgani, nel precedere la differenziazione organica, sia altresì irriducibile ad ogni configurazione psichica, con la conseguenza di farne una figura emblematica e paradigmatica di quell'esperienza del desiderio auspicata da Deleuze e Guattari.

Ma in che modo questi esempi di beatitudine concernerebbero la biopolitica? In merito, crediamo sia possibile individuare almeno due argomenti, entrambi sviluppati non da Deleuze in prima persona ma da alcuni pensatori che, per così dire, ne hanno ereditato la posta politica: Hardt e Negri, da un lato, il cui discorso sembra spingersi in direzione di una biopolitica affermativa; Esposito, dall'altro, la cui trattazione sembra culminare in una torsione vitalista del giuridico, che, come tale, assume i tratti di una specie di bio-diritto ${ }^{22}$.

\footnotetext{
${ }^{20}$ DELEUZE, G., GUATTARI, F. L’anti-Edipo. Capitalismo e schizofrenia. Torino: Einaudi, 2002, p. 29.

${ }^{21}$ Ivi, p. 30. Per una disamina dei ragionamenti alla base di questi approdi, cfr. MONTEBELLO, P. «L'instinct de mort chez Deleuze. La controverse avec la psychanalyse», doispontos, 8, (2011).

${ }^{22}$ A dire il vero il panorama politico e filosofico dischiuso dalla vulgata deleuziana sembra essere ben più vasto. In esso, difatti, si potrebbero includere: a) le bio-teo-politiche escatologiche (cfr. PERTICARI, P. «Pensare a termine. Jacob Taubes: un apprendimento concreto nel pensiero bio-teo-politico di questo tempo». In: AA. VV. Biopolitica minore. Roma: manifestolibri, 2003, pp. 67-84); b) il post-umanismo bio-tecnologico di Donna Haraway; c)
} 
Quanto alla biopolitica affermativa, Hardt e Negri sembrano fare lavorare l'implicito del discorso foucaultiano. Differentemente dal gesto di Foucault che ha per lo più considerato il biopotere dal lato del potere - ossia dell'entità che irretisce il bios nelle maglie della politica - i due pensatori lo pensano dal lato del bios, il quale, alla luce della riflessione sul piano d'immanenza di cui si è parlato in questo paragrafo, assurge a cifra mediante cui: a) svelare che la realtà sociale è una moltitudine di singolarità irriducibili ad ogni mediazione e rappresentazione univocizzante ed omologante (come lo sono state quelle tipiche del contrattualismo moderno); b) esplicitare le potenzialità del nuovo ordine mondiale, del cosiddetto Impero, il quale, pur sottomettendo i viventi mediante il lavoro immateriale, rischia di crollare per mano della capacità propria a questo lavoro, ossia per mano della capacità di portare alla luce le virtualità ancora inespresse della vita ${ }^{23}$; c) consente di fare leva sulla potenza di un desiderio che, in virtù della propria caoticità ed escandescenza, si presta a funzionare come fattore propulsore delle rivoluzioni sociali.

Venendo invece al concetto di biodiritto, si tratta, come nota Esposito, di considerare la filosofia deleuziana - ed in particolare alcuni dei suoi concetti (come quello di corpo senza organi o di desiderio presimbolico) - alla stregua di uno strumento utile a disinnescare il paradigma che, per secoli, ha presieduto al processo di attribuzione della tutela giuridica: il cosiddetto paradigma della persona. Stando a questo paradigma, il godimento dei diritti non conseguirebbe dal semplice fatto di essere in vita, bensì dal fatto di essere considerati alla stregua di persone $^{24}$, cosa che ha implicato la valorizzazione della persona e la

l'antropotecnica di Peter Sloterdijk, Per approfondimenti in merito, cfr. BAZZICALUPO, L. Biopolitica. Una mappa concettuale. Roma: Carocci, 2010; AA. VV., Resisting Biopolitics. Philosophical, Political, and Performatives Strategies. New York-London: Routledge, 2016.

${ }^{23}$ Così, ad esempio, si esprime Hardt: "Quando parliamo del lavoro come produttore del processo storico (e anche del processo storico capitalista), ne parliamo in termini di lavoro vivente ovvero di una forza che non è solo produzione, ma anche capacità di intervenire sulla riproduzione, sull'intelletto e sugli affetti, ecc. [...] a partire da questo risorgimento molto selvaggio del sapere e dell'azione, vedo una formidabile sorgente creativa della storia" (BONNAUD, I., GRELET, S., POTTE-BONNEVILLE, M., REVEL, J. «Il contro-impero attacca. Intervista a Toni Negri». In: AA. VV. Biopolitica Minore, cit., p. 222). Gli snodi principali di queste tesi si trovano in: HARDT, M., NEGRI, A. Impero. Il nuovo ordine della globalizzazione. Milano: Rizzoli, 2001). Per una disamina delle ricadute del paradigma deleuziano quanto al capitalismo, cfr. BAZZICALUPO, L. «Attualità politica di Deleuze», Etica \& Politica / Ethics \& Politics, 3, (2016), pp. 143-162.

\footnotetext{
${ }^{24}$ Secondo Esposito, il paradigma della persona sarebbe emerso per contrastare gli esiti terrificanti dell'eugenetica nazista, e, quindi, per colmare lo scarto tra vita e diritto, lo scarto in forza del quale solo il puro sangue ariano, e non il semplice fatto di vivere, permetteva il riconoscimento di una personalità giuridica. Il problema è che la categoria di persona, di tradizione romano-cristiana, dovendo rispondere all'eugenetica, ha prodotto un plusvalore rispetto al mero sostrato biologico con cui si è riconfermato lo scarto tra vita e diritto. La persona, infatti, costituisce una
} 
svalorizzazione della vita. Contro questa svalorizzazione (che testimonia lo statuto biogiuridico-negativo del paradigma personologico) Esposito sembra rintracciare nella filosofia deleuziana gli spunti per sviluppare un pensiero biogiuridico-affermativo. Se è vero infatti che ogni ente sia un'attualizzazione della virtualità immanente alla vita, altresì vero è, allora, che nessuna parte di essa sia tale da essere valorizzata a scapito di altre (come è accaduto, appunto, con la persona), "che nessuna parte di essa [...] [possa] essere distrutta a favore di un'altra” ${ }^{25}$.

Ecco dunque gli approdi bio-politici e bio-giuridici della beatitudine: "decostruiti gli schemi simbolici [...], si afferma come ultima parola l'affermatività, la positività del reale stesso o della vita nella sua pura pulsionalità" ${ }^{26}$.

\section{Biopolitica ed im-potere in Jacques Derrida}

Giunti a questo punto, ci volgiamo al pensiero derridiano al fine di esplicitare le ragioni che permettono di concepire la decostruzione alla stregua di un inedito pensiero del vivente mediante cui decostruire la biopolitica negativa (e, perché no, anche quella affermativa di cui si è parlato precedentemente).

\footnotetext{
sporgenza del lato razionale della soggettività; il criterio per l'attribuzione della personalità, ancora una volta, invece di essere la vita, è stato scorto nel suo plusvalore. In altri termini, se persona significa soggetto sovrano, ne consegue che la categoria dei diritti umani continua a restare qualcosa di profondamente discriminante: l'umano è tagliato fuori dalla persona che costituisce l'unico criterio di validazione con cui ottenere tutela giuridica. Dietro all'opzione eugenetica, come anche dietro a quella personalista, troviamo sempre il medesimo presupposto, lo zoon logon echon. Esso comporta la "gestione produttiva della vita, [...], nel primo caso a favore del corpo razziale del popolo eletto e nel secondo del soggetto individuale che ne diviene padrone" (ESPOSITO, R. Terza Persona, cit., p. 112). Per un maggiore dettaglio sulla questione del diritto tra Esposito e Deleuze, cfr: PEZZANO, G. «Stirner, Deleuze, Esposito: la maschera del diritto e il vitalismo anarchico», Lessico di etica pubblica, 2, (2010). Per una rielaborazione geopolitica del tema si veda: PÉREZ, M. G. «Roberto Esposito lector de Deleuze. De la biopolítica afirmativa y la communitas a la caosmunidad geopolítica», Bajo Palabra, Revista de Filosofía, 17, (2017).

${ }^{25}$ ESPOSITO, R. Bíos, cit., pp. 214-215. Considerando, inoltre, che il paradigma personologico è frutto, secondo Esposito, del paradigma della teologia politica, ne consegue che una filosofia dell'impersonale dovrebbe essere ciò che garantisce la liquidazione del teologico-politico; per dettagli in tal senso si veda R. Esposito, Due. La macchina della teologia politica e il posto del pensiero, Einaudi, Torino, 2013. Uno tra i più attenti studiosi del dispositivo biopolitico che ha mostrato alcune perplessità rispetto a queste ipotesi è, certamente, Giorgio Agamben. Per un approfondimento in merito, cfr. AGAMBEN, G. «L'immanenza assoluta». In: AGAMBEN, G. La potenza del pensiero. Vicenza: Neri Pozza Editore, 2005; per una indagine circa i rapporti tra Agamben e Deleuze, cfr. D’HOEST, F., LEWIS, T-E. «"Immanence: A Life...”: An Educational Formula?», Philosophy of education, (2015).

${ }^{26}$ BAZZICALUPO, L. Biopolitica, cit., p. 123. Per una riflessione generale su Deleuze e la questione biopolitica, cfr. ANTONELLI, M. «Gilles Deleuze y el debate biopolítico contemporáneo», Revista de Filosofía y Teoría Política, 46, (2015).
} 
Si tratta di un pensiero per la cui strutturazione del tutto significativo è stato il contributo della psicoanalisi (soprattutto freudiana $)^{27}$.

Un piccolo cenno a quella nozione psicoanalitica che, come nota Derrida, è alla base del ripensamento dell'idea di vita - ossia alla nozione di pulsione di appropriazione [Bemächtigungstrieb] - è d'obbligo.

In prima battuta, il concetto di pulsione di appropriazione viene menzionato da Freud nei Tre saggi sulla teoria sessuale al fine di indicare una particolare inclinazione psichica comune a tutti gli uomini, vale a dire la crudeltà. Più propriamente, Freud allude al "piacere per ogni specie di umiliazione e assoggettamento" ${ }^{28} \mathrm{O}$ "inclinazione alla sopraffazione" ${ }^{29} \mathrm{che}$, pur indipendente dalla sessualità, può "ad essa congiungersi per anastomosi” ${ }^{\circ}$, manifestandosi nelle pulsioni parziali tipiche della sessualità pregenitale, ovvero nella fase orale - con le fattezze di una pulsione d'“incorporazione" ${ }^{31}$ cannibalesca - e nella fase sadico-anale con i tratti, appunto, della "pulsione di appropriazione"32. Pulsioni e loro destini è la seconda opera ove Freud, in occasione di un'analisi relativa alla relazione fra sadismo e masochismo, definisce il Bemächtigungstrieb come un'istanza sadica - tale da prendere "in considerazione il fatto di arrecare dolore"33 - solo se, precedentemente, ha attraversato il masochismo tramite cui sperimentare l'equivalenza tra dolore e godimento. È tuttavia a partire da Al di là del principio di piacere, ovvero con l'introduzione del

\footnotetext{
${ }^{27}$ Come nel caso di Deleuze, anche per Derrida il panorama che ha presieduto al pensiero del vivente è ben più ampio, e comprende ancora una volta nomi di spicco della tradizione: Husserl, Hegel, Heidegger, Nietzsche, Lévinas. Evidentemente, l'approfondimento di questo panorama ci avrebbe preso troppo spazio. Si è scelto, dunque, di limitarsi a quella matrice la cui rilettura decostruttiva sembra avere una ricaduta biopolitica, per certi versi, più diretta: appunto la psicoanalisi freudiana. Ad ogni modo, per una ricostruzione circa il pensiero del vivente in Derrida, cfr. KELLY, C-J. «Trace, Dissemination, "Survivre": Derrida's "Biology" and the "Socius" », The international Journal of Humanities, 5; DI MARTINO, C. «Derrida e il pensiero del vivente», Epékeina. International Journal of Ontology, History and Critics, 1, (2012); REGAZZONI, S. Derrida. Biopolitica e democrazia. Genova: Il Melangolo, 2012; GERACI, S. «La questione del vivente: traccia, biologia, macchinalità». In: DALMASSO, G.. DI MARTINO, C., RESTA, C. L'a-venire di Derrida. Milano: Mimesis, 2014; VITALE, F. Biodeconstruction. Jacques Derrida and life science. Albany: SunyPress, 2018.

${ }^{28}$ FREUD, S. «Tre saggi sulla teoria sessuale». In: FREUD, S. Opere (1900-1905). Tre saggi sulla teoria sessuale e altri scritti, vol. 4. Torino: Boringhieri, 1970, p. 470.

${ }^{29}$ Ibidem.

${ }^{30}$ Ivi, p. 501.

${ }^{31}$ Ivi, p. 506

${ }^{32}$ Ibidem.

${ }^{33}$ FREUD, S. «Pulsioni e loro destini». In: FREUD, S. Opere (1915-1917). Introduzione alla psicoanalisi e altri scritti, vol. 8. Torino: Boringhieri, 1978, p. 24.
} 
concetto di pulsione di morte, che il Bemächtigungstrieb acquisisce tutta la sua pregnanza. In quest'opera, scritta dopo la prima guerra mondiale, Freud avanza l'ipotesi secondo cui la tendenza preponderante dello psichismo sia quella relativa alla neutralizzazione di ogni stato di tensione. Dal fatto che la tensione subentra a fronte di stati d'eccitazione inappagati, ne consegue che la neutralizzazione di cui si è detto implichi l'azzeramento di ogni stato di eccitazione, o, per dirla altrimenti, il compimento di una scarica che riporti il "livello [di Q认ं] a zero"34 (inorganico, Nirvana o Thanatos). In questo senso, tutte le pulsioni sono rilette da Freud come istanze regressive e conservatrici che spingono l'organismo al ripristino dello stato inorganico precedente alla vita:

Una pulsione sarebbe dunque una spinta, insita nell'organismo vivente, a ripristinare uno stato precedente al quale quest'essere vivente ha dovuto rinunciare sotto l'influsso di forze perturbatrici provenienti dall'esterno; sarebbe dunque una sorta di elasticità organica, o, se si preferisce, la manifestazione dell'inerzia che è propria alla vita organica ${ }^{35}$.

È fondendosi con quell'energia sottesa, stando a Freud, a tutti i viventi (vale a dire la libido [Eros]) che, detta elasticità organica assume i tratti della "pulsione di distruzione, pulsione di appropriazione, di volontà di potenza" ${ }^{6}$. Si evince, dunque, che, con Aldilà del principio di piacere, il Bemächtigungstrieb assume le fattezze di quell'istanza che spinge il vivente al ricongiungimento con sé, alla morte dalla quale proviene. Senonché, come nota Freud, l'organismo non vuole morire una morte qualunque, bensì una morte propria, la propria morte, il cui interesse è, per così dire, tutelato da un gruppo di pulsioni apparentemente avverse a quella appena menzionata, vale a dire le pulsioni di auto-conservazione:

Sono pulsioni parziali, che hanno la funzione di garantire che l'organismo possa dirigersi verso la morte per la propria via tenendo lontane altre possibilità di ritorno all'inorganico che non siano quelle immanenti allo stesso organismo. Non dobbiamo più contare sulla misteriosa tendenza dell'organismo (così difficile da inserire in qualsiasi contesto) ad

\footnotetext{
${ }^{34}$ FREUD, S. «Progetto di una psicologia». In: FREUD, S. Opere (1892-1899). Progetto di una psicologia e altri scritti, vol. 2. Torino: Boringhieri, 1968, p. 203.

${ }^{35}$ FREUD, S. «Al di là del principio di piacere». In: FREUD, S. Opere 1917-1923. L'Io e l'Es e altri scritti, vol. 9. Torino: Boringhieri, 1977, p. 222.

${ }^{36}$ FREUD, S. «Il problema economico del masochismo». In: FREUD, S. Opere (1924-1929). Inibizione, sintomo e angoscia e altri scritti, vol. 10. Torino: Boringhieri, 1978, p. 9.
} 
affermarsi contro tutto e tutti. Essa si riduce al fatto che l'organismo vuole morire solo alla propria maniera. Anche questi custodi della vita sono stati in origine guardie del corpo della morte. Si determina così il paradosso che l'organismo vivente si oppone con estrema energia a eventi e (pericoli) che potrebbero aiutarlo a raggiungere più in fretta lo scopo della sua vita $^{37}$.

A fronte di questo paradosso (da Freud immediatamente scartato subito dopo averlo ipotizzato), si inserisce la lettura derridiana. Riprendendo questo passaggio, secondo Derrida, diventa possibile esplicitare il fatto che la vita non sia caratterizzata da due spinte tra loro antagoniste - la pulsione di morte e la pulsione di vita - poiché, al contrario, consiste in un'unica spinta [Trieb], la pulsione del proprio o di appropriazione:

La pulsione del proprio sarebbe più forte della vita $e$ della morte. Occorre allora esplicitare le implicazioni di tale enunciato. Se, autoteleguidando il proprio lascito, la pulsione del proprio è più forte della vita e più forte della morte, il fatto è che, senza appartenere alla vita o alla morte, la sua forza non la qualifica altrimenti che attraverso la propria pulsività e che questa pulsività sarebbe lo strano rapporto a sé che si definisce rapporto al proprio: la più pulsiva delle pulsioni è la pulsione del proprio, in altri termini quella che tende a riappropriarsi. Il movimento di riappropriazione è la pulsione più pulsiva. Il proprio della pulsività è il movimento o la forza di riappropriazione. Il proprio è la tendenza ad appropriarsi $^{38}$.

Dire che la pulsione di appropriazione precede l'opposizione tra vita e morte, significa esplicitare che tali due spinte [Trieb] altro non siano che una specifica torsione della medesima pulsione. Se è infatti vero che l'inorganico (o morte) è l'origine il cui ripristino garantisce l'appropriazione di sé, è altresì vero che, per appropriarsi di sé (e quindi morire una morte propria), sia necessario imparare a controllare la tendenza alla scarica nirvanica convertendola nella cosiddetta pulsione di vita.

Ma se così stanno le cose, se la vita non è altro che una spinta [Trieb] verso una meta (la morte) che, nel momento in cui viene

\footnotetext{
${ }^{37}$ FREUD, S. «Al di là del principio del piacere», cit., p. 225 .

${ }^{38}$ DERRIDA, J. Speculare - su "Freud”. Milano: Raffaello Cortina, 200o, p. 115.
} 
avvicinata, deve essere pure allontanata (altrimenti a venire meno è la possibilità medesima di mettersi sulle sue tracce), allora ne consegue che uno dei termini più adeguati per designare il fenomeno "vita" sia quello di aporia. Dal greco, aporos è un termine composto da poros, che significa cammino, e dalla "alfa” privativa, la cui funzione consiste nell'esplicitare lo scacco intrinseco al cammino di cui si sta parlando:

\begin{abstract}
Ne consegue inoltre che l'Umweg non è una specie derivata di cammino o di passo. Non è una determinazione di passaggio, una definizione più stretta o più rigorosa del passaggio, è il passaggio. (Il) Weg (è) Umweg fin dal primo passo del passo [pas du pas]. E ricordiamo di passaggio: weg, l'avverbio, significa parimenti "via". Si può intenderlo come un ordine, una richiesta o un desiderio: fort! $\mathrm{Via}^{39}$ !
\end{abstract}

Che si utilizzi il termine greco aporos, il termine tedesco Umweg, o quello francese pas (adoperato da Derrida in svariate occasioni sempre al fine di affrontare la questione oggetto del presente paragrafo), la sostanza delle cose non cambia, o quanto meno non cambia in maniera radicale. Designando infatti una spinta che si compie mentre si nega, questi termini mostrano che il carattere specifico della vita, intesa quale istanza di appropriazione di sé, sia l'espropriazione:

Ogni volta, con la pulsione, la forza o il movimento, la tendenza o il telos, bisogna mantenere uno scarto. Il che interdice alla pulsione del proprio d'essere designata attraverso un'espressione pleonastica che definisca il semplice rapporto a sé dell'interiorità [...]. Il proprio non è il proprio, e se si appropria, il fatto è che si espropria - propriamente, impropriamente. La vita la morte non si oppongono più ${ }^{40}$.

La vita e la morte non si oppongono più nella misura in cui il solo modo di cercare la morte (e quindi di appropriarsi di sé) è quello di restare in vita, il che differendo l'istanza stessa di appropriazione, ragion per cui, più che di espropriazione, si dovrebbe, come fa Derrida, ricorrere ad un neologismo, ossia al termine di ex-appropriazione, il quale, reciprocando appropriazione ed espropriazione, ben si presta a significare il carattere aporetico del pulsionale:

\footnotetext{
${ }^{39}$ Ivi, p. 113 .

$4^{\circ}$ Ivi, p. 115 .
} 
Niente più nome proprio, nessun nome proprio che non si chiami o non si richiami a questa legge dell'oikos. Nella salvaguardia del proprio, al di là dell'opposizione vita morte, il suo privilegio è anche la sua vulnerabilità, si può anzi dire la sua improprietà essenziale, l'es-appropriazione [exappropriation] (Enteignis) che lo costituisce ${ }^{41}$.

Se nell'opera in cui è stata introdotta, vale a dire in Speculare su "Freud", detta improprietà, o meglio ex-appropriazione, è servita a decostruire il dualismo pulsionale freudiano, è, tuttavia, a partire da Stati d'animo della psicoanalisi, che essa è divenuta punto di partenza per una disamina delle ricadute politiche scaturenti dalla riconsiderazione derridiana del freudismo ${ }^{42}$.

Ammettere, con e contro Freud, che la vita sia un'istanza di exappropriazione, ossia un'istanza che si espropria mentre si appropria e viceversa, significa, concludere, ancora una volta con e contro Freud, che la mitigazione della spinta mortifera e distruttiva non dipenda da una contro-forza ad essa esteriore, ossia dalla pulsione di vita. Consegue da ciò che la strategia considerata dal padre della psicoanalisi essenziale al fine di assicurare la coesistenza sociale (la strategia consistente nell'imporre alla pulsione di morte delle soddisfazioni indirette contrapponendole la pulsione di vita ${ }^{43}$ ), altro non è che la traccia del differire della pulsione di appropriazione.

Il ragionamento freudiano sull'indiretto (cui si è appena fatto cenno), in questo senso, andrebbe riconsiderato come scaturigine dell'exappropriazione pulsionale della vita, al punto che, riprendendo le parole del filosofo franco-algerino: "il concetto dell'indiretto mi sembra tener conto, nella mediazione della diversione, di una discontinuità radicale, di

\footnotetext{
${ }^{41}$ Ivi, p. 119.

${ }^{42}$ Per ulteriori spunti in merito, cfr. MANSFIELD, N. The God Who Deconstruct Himself: Sovereignty and Subjectivity Between Freud, Bataille and Derrida. New York: Fordham University Press, 2010; GERACI, S. «Pulsione di crudeltà: Derrida e la psicoanalisi». In: AA. VV., L'evento dell'ospitalità tra etica, politica e geofilosofia. Per Caterina Resta. Milano: Mimesis, 2013.

${ }^{43}$ La strategia dell'indiretto è subentrata nel discorso psicoanalitico per via del fatto che Freud è sempre stato ben conscio dei pericoli che subentrerebbero nel caso in cui ci si abbandonasse ad un appagamento diretto della tendenza all'azzeramento energetico (Thanatos): al fine di contrastare questa prospettiva, la quale condurrebbe dritti al bellum omnium contra omnes, il padre della psicoanalisi ha mostrato come sia auspicabile opporre alla suddetta tendenza le pulsioni di vita, le quali, per mano soprattutto delle pulsioni di auto-conservazione, educano gli individui a dilazionare il passaggio all'atto (scarica).
} 
un'eterogeneità $[\ldots]$ che nessun sapere psicoanalitico in quanto tale potrebbe autorizzare" 44 .

È grazie alla rielaborazione della nozione freudiana di diversione vitale, che la decostruzione diventa foriera di un ripensamento del potere.

Per comprendere come l'indirezione vitale concerne il potere va, tuttavia, fatta una premessa. Per Derrida, la vita, o meglio la pulsione di appropriazione, costituisce il fulcro germinativo di tutti i poteri. Una conferma la si ritrova nell'etimologia. Nella maggior parte delle lingue indoeuropee, difatti, la radice del termine 'potere' rinvia ad un movimento, al movimento di appropriazione di sét5. Il che non è senza ragione: è ovvio che l'esercizio del potere implica un potere di e su se stessi. Senza padronanza di sé, l'esercizio del potere diventa impossibile, ragion per cui il movimento di auto-appropriazione pulsionale finisce per corrispondere all'archetipo quasi-trascendentale di tutti i poteri. Per dirla a rovescio, si tratta di ammettere che tutti i poteri altro non sono che traduzioni ed articolazioni della medesima pulsione, della pulsione di appropriazione.

Posto che la pulsione di appropriazione sia il cuore di tutti poteri, e posto che l'indiretto, e quindi l'ex-appropriazione, sia il cuore della pulsione di appropriazione, ne consegue che ad essere ex-appropriato sia il potere stesso, il quale, in ragion dell'ex-appropriazione di cui si è appena detto, scopre un'impotenza intrinseca alla propria facoltà di potere. Si tratta di un im-potere derivante, in prima istanza, dal fatto di non potere appropriarsi di sé, e, perciò stesso, di non potere esercitare il proprio potere sugli altri, cosa che, ben lungi dal neutralizzare l'istanza di potere, al contrario, la rilancia esacerbandola (è a causa di tale non-potere che il potere diventa oggetto di un'infinita ed insaziabile bramosia).

Al fine di tratteggiare le caratteristiche di questo im-potere, Derrida introduce un concetto inedito di forza. Un concetto di forza mediante il quale eccedere la nozione di forza a cui siamo abituati (la forza del potere). All'opposto di quest'ultima, la quale opera attualizzando le facoltà che pertengono al potere di cui si dispone, la forza dell'im-potere è una "forza debole", la quale, facendosi carico dell'ex-appropriazione (o im-potere) consustanziale al proprio potere, lascia accadere le possibilità che dall'exappropriazione in questione scaturiscono. Ma quali sono queste

\footnotetext{
44 DERRIDA, J. Stati d'animo della psicoanalisi. L'impossibile aldilà di una sovrana crudeltà. Pisa: ETS, 2013, p. 62.

${ }^{45}$ BENVENISTE, E. Vocabolario delle istituzioni indoeuropee I. Economia, parentela, società. Torino: Einaudi, 2001.
} 
possibilità? La risposta a questa domanda dipende dal fatto che l'exappropriazione è una specie di resa vulnerabile ed incondizionata all'evento. È evidente, perciò, che ad essere in gioco sono delle possibilità che non si lasciano programmare né pensare preventivamente. Non si tratta, cioè, di quelle virtualità deducibili dalle facoltà di cui il potere dispone, ma, al contrario, dell'imprevedibilità, e finanche dell'impossibilità, che l'esposizione vulnerabile lascia accadere. Ciò detto, differentemente dalla "forza del potere", la "forza debole o dell'im-potere" è un'istanza paradossale: è il cosiddetto potere dell'impossibile. "Senza potere ma senza debolezza. Senza potere ma non senza forza, foss'anche una certa forza della debolezza" ${ }^{46}$, questa forza debole è in grado di sopportare il proprio im-potere, ovvero di lasciare accadere l'impossibile:

Ciò che sto nominando definisce l'economia del possibile e del potere, dell' «io posso». Ne va in effetti dell'economia in tutti i sensi del termine, quella della legge del proprio (oikonomia) e della domesticità familiare, quella dello Stato sovrano, del diritto di proprietà, del mercato, del capitale, dei modi di appropriazione in generale, e più di tutto ancora, di ciò che Freud chiama l'economia psichica. Mi richiamo qui a un aldilà dell'economia, quindi dell'appropriabile e del possibile ${ }^{47}$.

Proprio quest'aldilà dell'economia del possibile costituisce il punto da cui ripartire per pensare il biopolitico. Se, come ha detto Agamben, quest'ultimo scaturisce dalla struttura eccezionale del potere (che ha conferito ai politici una competenza sulla vita e sulla morte ${ }^{48}$ ), l'im-potere è la messa in discussione di tutto ciò. In un certo senso, l'im-potere può essere considerato come un'eccezione dell'eccezione. Svelare l'im-potere del potere, l'esposizione alla quale tutti i poteri sono esposti nonostante

\footnotetext{
${ }^{46}$ DERRIDA, J. Incondizionalità o sovranità. L’Università alle frontiere dell'Europa. Milano: Mimesis, 2006, p. 44. Come afferma Caterina Resta: "Questa «forza senza potere» e vulnerabile senza condizione e senza difese, questo salutare abbassamento delle difese immunitarie, che è anche un indebolimento della sovranità dell'io impone di «arrendersi» all'altro, in sé-fuori-di-sé [...]. Sarà allora necessario pensare l'incondizionato non dalla parte del potere e del fantasma di una sovranità autoreferenziale che si auto-determina e si auto-pone, ma dal lato di una ipseità vulnerabile che, esponendosi senza potere, costringe a pensare l'im-potere, l'im-possibile come esposizione all'evento dell'altro. Una ipseità che, senza difese, più passiva di ogni passività, si espone, infinitamente vulnerabile, alla carezza e alla ferita dell'altro, fino a lasciarsi «abitare» dall'altro, a sop-portarlo dentro di sé” (RESTA, C. «Un'esposizione vulnerabile», Phasis. European Journal of Philosophy, o, (2012), p. 16o). Per una trattazione approfondita di questo tema si veda C. Resta, L'impossibile, il non potere, in Id. La passione dell'impossibile. Saggi su Derrida, Genova, Il Melangolo, 2016.

${ }^{47}$ DERRIDA, J. Stati d'animo della psicoanalisi, cit., p. 65 .

${ }^{4}{ }^{8} \mathrm{Cfr}$. AGAMBEN, G. Homo sacer. Il potere sovrano e la nuda vita. Torino: Einaudi, 2005.
} 
tutto, implica una riconsiderazione di quel bios che per Foucault abbiamo visto costituire il polo passivo del dispositivo biopolitico. Lungi dall'essere un oggetto decidibile politicamente, esso assume i tratti dell'imprevedibile cui tutti i poteri sono esposti, è l'impotere del potere, ciò che, dunque, eccepisce l'eccezione della sovranità e, perciò stesso, ne impedisce la torsione biopolitica. Ad essere eccepiti sono, addirittura, i due cardini fondamentali della biopolitica: l'anatomo-politica dei corpi (ossia le politiche di promozione della vita) e la biopolitica delle popolazioni (ossia l'assembramento di individui in virtù di precise fattezze geneticobiologiche). Anatomo-politica del corpo e biopolitica delle popolazioni, non trovano forse un limite nel proprio oggetto - il bios - il quale, prima di essere la sede delle decisioni politiche, assurge ad imprevedibile a cui ogni potere è esposto sin nel proprio intimo?

Quanto detto, ha, inoltre, pesanti conseguenze in materia di biopolitica affermativa. Se l'oggetto del biopotere - il bios - svela l'impossibile al cuore di tutti i poteri, su che basi considerare detto bios alla stregua di una contro-potenza o meglio di una potenzialità virtuale? Questa potenzialità virtuale, agli occhi della decostruzione, non è forse un altro nome di quella bramosia che subentra nel momento in cui il potere non accetta la propria ex-appropriazione rispetto a se stesso? La propria vulnerabilità? La propria apertura all'impossibile, all'incalcolabile ed all'imprevedibile? Non è forse lecito rintracciare tali sospetti in molteplici punti dell'opera derridiana, per esempio in La bestia e il sovrano I, e, più precisamente, nella dodicesima séance, quando, sulla scorta di Heidegger, Derrida manifesta le proprie reticenze nei confronti di ogni vitalismo, dicendo di scorgere in quest'ultimo "molti nemici" 49 ? A cosa e a chi allude Derrida? Chi sono questi nemici?

Purtroppo è difficile dare risposte certe. Derrida ha in mente qualcuno di cui non svela l'identità, mantiene il suo gusto del segreto.

Probabile che nel mirino derridiano vi siano i sostenitori di quel pensiero pre-individuale oggi noto come biopolitica affermativa.

E non è forse quella appena menzionata una delle poste in gioco della riflessione derridiana circa il corpo-senza-organi di Artaud, che, non a caso, Derrida ha concepito come una specie di delirio d'appropriazione mediante cui sconfiggere la formattazione della vita operata dalla 
repressione razionalistica moderna ${ }^{50}$ ? E che dire, ancora, dell'esplicita requisitoria elaborata da Derrida in Toccare, Jean-Luc Nancy, ove proprio il corpo senza organi viene giudicato (alla luce delle rielaborazioni di Deleuze e Guattari) come "postulazione continuista» ${ }^{1}$ che, nell'appianare tutte le striature dell'esperienza, tutti gli scarti, mira a un'appropriazione piena e totale del proprio godimento, assumendo, come tale, i tratti di un «fantasma metafisico [...] cristiano" ${ }^{2}$ ?

I casi appena riportati, non sono forse suscettibili di essere considerati come l'indice dei dubbi che un pensiero di matrice decostruttiva non può non avere rispetto alla cosiddetta biopolitica affermativa ${ }^{53}$ ?

\section{L'avvenire della biopolitica al bivio della filosofia della differenza}

Alla luce di quanto detto fino ad ora, riteniamo opportuno formalizzare la seguente conclusione: la filosofia francese della differenza rappresenta per la biopolitica una specie di bivio, un bivio fondamentale il cui intraprendimento avrà delle conseguenze di notevole importanza.

La ragione per cui si è scelto di parlare di bivio è presto detta: gli autori oggetto di analisi in questa sede, sembrano giungere a dei nodi problematici comuni - l'idea di desiderio e la coppia possibile/impossibile - che, tuttavia, affrontano e approcciano compiendo percorsi diametralmente opposti.

Cominciamo con il desiderio. Se in un caso, nel caso deleuziano, ma sarebbe più corretto dire deleuziano-guattariano, prevale l'idea di un "desiderio [che] non manca di nulla" ${ }^{54}$, e che, come tale, è capace di un godimento completo, pieno e del tutto privo di resti, nell'altro caso, nel caso derridiano, prevale l'idea di un desiderio ex-appropriato, afflitto, a

\footnotetext{
${ }^{50}$ In questo senso, il gesto di Artaud, pur professandosi del tutto contrario a quello della tradizione metafisica, finisce dunque per ripeterne il movimento generale. Come essa, infatti, insegue il "sogno di una vita senza differenza" (DERRIDA, J. La scrittura e la differenza. Torino: Einaudi, 2002, p. 232) con la variante per cui "la soteriologia sarà l'escatologia del corpo proprio" (Ivi, p. 237) invece dell'auto-appropriazione razionale di se stessi.

${ }^{51}$ DERRIDA, J. Le toucher, Jean-Luc Nancy. Paris : Galilée, 2000, p. 143.

${ }^{52}$ Ivi, p. 144

${ }^{53}$ Per ulteriori approfondimenti quanto alle ricadute della decostruzione in ordine sia alla biopolitica negativa, sia a quella affermativa, cfr. BISET, E. «Biopolítica, soberanía y deconstrucción», en THÉMATA. Revista de Filosofía, 56, (2017).

${ }^{54}$ DELEUZE, G., GUATTARDI, F. L’anti-Edipo, cit., p. 29.
} 
causa di questa ex-appropriazione, da una mancanza che, nell'impedire ogni azzeramento energetico [Thanatos], funge da unica garante del godimento, finendo per acquisire i tratti di una mancanza-senzamancanza, o, per dirla con Derrida, i tratti di un "senza senza senza"55.

Venendo alla questione del possibile nei suoi rapporti all'impossibile, si tratta dell'approdo relativo alle appena menzionate concezioni del desiderio. Mentre il desiderio deleuziano-guattariano si caratterizza per il fatto di rendere tutto possibile, e quindi di realizzare l'impossibile, il desiderio derridiano è ben lungi dal realizzare l'impossibile poiché, al contrario, lo lascia accadere.

È abbastanza evidente, in questo senso, che la filosofia francese della differenza approccia il tema della vita in maniera tale da imporre una scelta, una strada invece dell'altra, la strada di una vita che realizza l'impossibile e la strada di una vita impossibile.

Sebbene si corra il rischio di semplificare troppo le cose, riteniamo, comunque auspicabile, al punto di estremizzare il risultato del nostro lavoro comparativo, riconsiderare le due prospettive qui studiate come in atto di denunciarsi reciprocamente: se è vero che la vita costituisce la linfa emergenziale del potere, allora la filosofia derridiana dell'im-potere sembra mostrare la totale inefficacia delle biopolitiche affermative: opporsi al potere mediante la potenza vitale non significa alimentare quella bramosia che il potere matura a causa della propria exappropriazione? D'altro canto, sulla base del ragionamento biopoliticoaffermativo, non sembra lecito credere che l'im-potere derridiano, con il suo scetticismo nei confronti della possibilità di elevare l'energia vitale a fonte dei gesti rivoluzionari, rischia di affievolire la capacità di lotta delle forze sociali in campo, finendo per agevolare lo status quo?

Se da un lato, sembra profilarsi il rischio di un vitalismo politico destinato a sprofondare nel fantasma che pure tenta di neutralizzare ${ }^{56}$, nel fantasma del potere; dall'altro, a fare capolino, sembra essere il pericolo di

\footnotetext{
55 DERRIDA, J. Speculare su - “Freud”, cit., p. 168.

${ }^{5}$ Per approfondimenti sul tema si veda: RESTA, C. Bio-thanato-politica: una questione di vita "e" di morte. In: STIMILLI, E. Decostruzione o biopolitica?, Macerata: Quodlibet, 2017. Queste inquietudini erano anche quelle di Lacan, il quale, in proposito, così si esprime: "Ciò a cui aspirate, come rivoluzionari, è un padrone. L'avrete" (LACAN, J. Il seminario. Libro XVII. Il rovescio della psicoanalisi. 1969-1970. Torino: Einaudi, 2001, p. 259).
} 
quella nichilistica rassegnazione che secondo alcuni si cela nella nozione derridiana di im-potere ${ }^{57}$.

Prendere parte a questa diatriba, parteggiarvi, in definitiva, significa dirigere la biopolitica verso due gesti che, più che paralleli ${ }^{58}$, sembrano l'inverso l'uno dell'altro: da un lato il gesto di "investire il campo sociale in forme inconsce, e quindi allucinare tutta la storia, delirare le civiltà, i continenti e le razze, e «sentire» intensamente un divenire mondiale"59; dall'altro il gesto di promuovere una forza che "si manifesta come rinuncia infinita alla forza, nell'interruzione assoluta della forza ad opera del 'senza forza" ${ }^{\text {"6o. }}$.

\section{Endereço postal:}

Faculdade de direito da USP

Largo São Francisco, 95 - Centro, São Paulo - SP, Brasil

Data de recebimento: 14/03/2018

Data de aceite: 17/09/2018

\footnotetext{
${ }^{57}$ Per un'indagine attorno a tali questioni, cfr. ESPOSITO, R. «Comunità, immunità, biopolitica». In: AA. VV. Spettri di Derrida, Genova: Il Melangolo, 2010; ESPOSITO, R. «Decostruzione o biopolitica». In: STIMILLI, E. Decostruzione o biopolitica?, cit., pp. 13-24. Per una ricostruzione critica della rilettura che Esposito propone quanto alla relazione tra decostruzione e biopolitica, cfr. MARCHENTE, A. «Autoimmunità: tra biopolitica e decostruzione», Esercizi filosofici, 9, (2014); LEWIS, M. Of (Auto-)Immune Life: Derrida, Esposito, Agamben, in AA. VV. Medicine and Society, New Perspective in Continental Philosophy, Springer, 2015.

${ }^{58}$ NANCY, J-L. Le differenze parallele. Deleuze e Derrida. Verona: Ombre Corte, 2008.

${ }^{59}$ DELEUZE, G., GUATTARI, F. L'anti-Edipo, cit., p. 109.

${ }^{60}$ DERRIDA, J. «Louis Marin. A forza di lutto». In: DERRIDA, J. Ogni volta unica, la fine del mondo. Milano: Jaca Book, 2005, p. 164 .
} 